\title{
Optimal stability of the Lagrange formula and conditioning of the Newton formula *
}

\author{
J.M. Carnicer, Y. Khiar, J.M. Peña \\ Departamento de Matemática Aplicada/IUMA \\ Universidad de Zaragoza, Spain
}

\begin{abstract}
A pointwise condition number associated to a representation of an interpolation operator is introduced. It is proved that the Lagrange formula is optimal with respect to this conditioning. For other representations of the interpolation operator, an upper bound for the conditioning is derived. A quantitative measure in terms of the Skeel condition number is used to compare the conditioning with the Lagrange representation. The conditioning of the Newton representation is considered for increasing nodes and for nodes in Leja order. For the polynomial Newton formula with $n+1$ equidistant nodes in increasing order, it is proved that $3^{n}$ is the best uniform bound of its conditioning and it is attained at the last node. Numerical experiments are included.
\end{abstract}

Keywords: Skeel condition number; Lagrange interpolation formula; Newton interpolation formula; Leja order 2010 MSC: 41A05; 41A10; 65D05

\section{Introduction}

Several authors have made comparisons between the Lagrange and the Newton formula for polynomial interpolation. In [15] (see also Chapter 5 and

*This work has been partially supported by the Spanish Research Grant MTM201565433-P (MINECO/FEDER), by Gobierno the Aragón and Fondo Social Europeo. 
Appendix of [16]), Trefethen suggests that the recommendation of some textbooks to use Newton interpolation formulae rather than Lagrange formulae is questionable. A fact that suggests a worse behaviour of the Newton formula with respect to the Lagrange formula is its dependence on the order of the nodes. Numerical experiments indicate that for large values of the degree most orders present numerical instability (see Section 3 of [1]). This instability has also been confirmed in [6], in particular at one end of the interval when the nodes are increasing or decreasing. According to [12], a Leja ordering can be used to reduce such instability. In this paper we present a quantitative measure of the instability of the Newton formula with respect to the Lagrange formula. For this purpose, we introduce a conditioning associated to a representation of the interpolant, which provides a pointwise measure of its stability. In the particular case of the Lagrange interpolation, this conditioning coincides with the usual Lebesgue function. In general, this conditioning plays for interpolation a similar role to that of the conditioning introduced by Farouki and Rajan (see [4]) for the evaluation of a function expressed in terms of a given basis. Optimal bases with respect to the Farouki-Rajan conditioning have been studied in several papers (see [3], [9], [11], [10], [8]). We prove in Section 2 of this paper the optimal stability of the Lagrange representation with respect to this new conditioning.

Another common fact shared by the two conditionings previously mentioned is the role played by the matricial Skeel condition number. The Skeel condition number $\operatorname{Cond}(A)$ of a matrix $A$ (see Section 7.2 of [5]) is always less than or equal to its classical condition number $\kappa_{\infty}(A)$, and so it provides more realistic bounds. In [8] it was shown that the Farouki-Rajan conditioning presents for the problem of function evaluations many analogies with the Skeel condition number of a matrix. In Section 2 of this paper, we also prove that the Skeel condition number of the inverse of the collocation matrix of a basis provides a bound for the worsening of the conditioning with respect to the Lagrange representation. Moreover, such a bound is attained at one of the nodes.

Section 3 is devoted to the analysis of the stability of the Newton representation compared with the Lagrange representation. It is shown that the maximum conditioning of the Newton representation coincides with the Skeel condition number of the inverse of the collocation matrix of the Newton basis.

Section 4 considers the Newton interpolation formula with nodes following a Leja ordering. We provide an upper bound of the ratio between the 
conditioning of the Newton representation with nodes following a Leja ordering and the conditioning of the Lagrange representation.

Section 5 and Section 6 show that $3^{n}$ is the best uniform bound for the conditioning of the Newton representation with $n+1$ equidistant points $x_{0}, \ldots, x_{n}$ in increasing order, and that it is attained at $x_{n}$. This bound is higher than the maximum value of the Lebesgue function, which is bounded above by $2^{n}$. Finally, numerical examples in Section 7 illustrate the results.

\section{Optimal stability of the Lagrange represen- tation}

The Lagrange interpolation problem on an $(n+1)$-dimensional subspace $U$ of $C[a, b]$ for a set of distinct nodes $x_{0}, \ldots, x_{n}$ in $[a, b]$ consists of finding $u \in U$ such that $u\left(x_{i}\right)=f\left(x_{i}\right), i=0, \ldots, n$, for a given function $f \in C[a, b]$.

If the Lagrange interpolation problem has a unique solution, we can define the Lagrange fundamental functions $l_{i} \in U$ by

$$
l_{i}\left(x_{j}\right)=\delta_{i j}, \quad i, j=0, \ldots, n,
$$

where $\delta_{i j}$ is the Kronecker symbol. The solution of the Lagrange interpolation problem can be expressed by the Lagrange formula $\sum_{i=0}^{n} f\left(x_{i}\right) l_{i}(x)$. Let us define the Lagrange interpolation operator

$$
L[f](x):=\sum_{i=0}^{n} f\left(x_{i}\right) l_{i}(x) .
$$

If we define the evaluation functionals

$$
\lambda_{i} f:=f\left(x_{i}\right), \quad i=0, \ldots, n,
$$

we obtain the Lagrange representation $L[f]=\sum_{i=0}^{n} \lambda_{i} f l_{i}$.

Let us to study different representations of the interpolation operator

$$
L[f]=\sum_{i=0}^{n} \beta_{i} f v_{i},
$$

where $\beta_{0}, \ldots, \beta_{n}$ belong to the space generated by the evaluation functionals $\lambda_{0}, \ldots, \lambda_{n}$ and $\left(v_{0}, \ldots, v_{n}\right)$ is a basis of $U$. We remark that the basis 
$\left(v_{0}, \ldots, v_{n}\right)$ is determined by the basis of functionals $\left(\beta_{0}, \ldots, \beta_{n}\right)$ since both bases form a dual pair, that is,

$$
\beta_{i} v_{j}=\delta_{i j}, \quad i, j=0, \ldots, n .
$$

We shall deal with the norm of interpolation operators

$$
\|L\|_{\infty}=\sup _{\|f\|_{\infty}=1}|L[f]|
$$

and the norm of functionals used for the representation

$$
\left\|\beta_{i}\right\|_{\infty}=\sup _{\|f\|_{\infty}=1}\left|\beta_{i} f\right|, \quad i=0, \ldots, n
$$

Let us recall that the Lebesgue function $\lambda(x)$ is defined as

$$
\lambda(x):=\sum_{i=0}^{n}\left|l_{i}(x)\right| .
$$

Its maximum value is called the Lebesgue constant. We shall use the following result (cf. Chapter 2 of [2]).

Theorem 1 The norm of the Lagrange interpolation operator is the Lebesgue constant, that is

$$
\|L\|_{\infty}=\max _{x \in[a, b]} \sum_{i=0}^{n}\left|l_{i}(x)\right| .
$$

Let us define the conditioning associated to the representation $L[f]=\sum_{i=0}^{n} \beta_{i} f v_{i}$ by

$$
\operatorname{cond}(x ; \beta):=\sum_{i=0}^{n}\left\|\beta_{i}\right\|_{\infty}\left|v_{i}(x)\right|
$$

The conditioning $\operatorname{cond}(x ; \beta)$ is a pointwise measure of the stability of the representation. In fact, if $\hat{f}$ is an approximation of $f$ then we have the bound

$$
|L[f](x)-L[\hat{f}](x)| \leq \sum_{i=0}^{n}\left|\beta_{i}[f-\hat{f}]\right|\left|v_{i}(x)\right| \leq\|f-\hat{f}\|_{\infty} \operatorname{cond}(x ; \beta) .
$$


Let us remark that the conditioning of the Lagrange representation corresponding to the evaluation functionals $\lambda_{0}, \ldots, \lambda_{n}$ coincides with the Lebesgue function

$$
\operatorname{cond}(x ; \lambda)=\sum_{i=0}^{n}\left|l_{i}(x)\right|=\lambda(x)
$$

because the norm of the evaluation functionals $\left\|\lambda_{i}\right\|_{\infty}=1, i=0, \ldots, n$. The following result provides the norm of a more general class of functionals.

Proposition 2 Let $\beta: C[a, b] \rightarrow \mathbb{R}$ be a functional of the form $\beta f=\sum_{i=0}^{n} c_{i} f\left(x_{i}\right)$. Then $\|\beta\|_{\infty}=\sum_{i=0}^{n}\left|c_{i}\right|$.

Proof: Observe that

$$
\|\beta\|_{\infty}=\sup _{\|f\|_{\infty}=1}\left|\sum_{i=0}^{n} c_{i} f\left(x_{i}\right)\right| \leq \sup _{\|f\|_{\infty}=1} \sum_{i=0}^{n}\left|c_{i}\right|\left|f\left(x_{i}\right)\right| \leq \sum_{i=0}^{n}\left|c_{i}\right| .
$$

Taking any continuous function $f$ such that $f\left(x_{i}\right)=\operatorname{sign}\left(c_{i}\right), i=0, \ldots, n$, we have the other inequality

$$
\sup _{\|f\|_{\infty}=1}\left|\sum_{i=0}^{n} c_{i} f\left(x_{i}\right)\right| \geq\left|\sum_{i=0}^{n} c_{i} \operatorname{sign}\left(c_{i}\right)\right|=\sum_{i=0}^{n}\left|c_{i}\right| .
$$

Therefore, the bound is attained and the supremum is a maximum.

Given a matrix $A=\left(a_{i j}\right)_{i, j=0, \ldots, n}$, we denote by $|A|:=\left(\left|a_{i j}\right|\right)_{i, j=0, \ldots, n}$ the matrix whose entries are the absolute values of the corresponding entries of A.

Proposition 3 Let $L[f]=\sum_{i=0}^{n} \beta_{i} f v_{i}$ be a representation of the Lagrange interpolation operator (1) and let $M:=\left(v_{j}\left(x_{i}\right)\right)_{i, j=0, \ldots, n}$ be the collocation matrix of the basis $\left(v_{0}, \ldots, v_{n}\right)$. Then

$$
\left(\begin{array}{c}
\beta_{0} \\
\vdots \\
\beta_{n}
\end{array}\right)=M^{-1}\left(\begin{array}{c}
\lambda_{0} \\
\vdots \\
\lambda_{n}
\end{array}\right) \quad \text { and } \quad\left(\begin{array}{c}
\left\|\beta_{0}\right\|_{\infty} \\
\vdots \\
\left\|\beta_{n}\right\|_{\infty}
\end{array}\right)=\left|M^{-1}\right| e,
$$

where $e:=(1, \ldots, 1)^{T}$. 
Proof: Since $f$ and $L[f]$ coincide at the nodes $x_{0}, \ldots, x_{n}$ we have,

$$
\lambda_{i} f=\lambda_{i} L[f]=\sum_{j=0}^{n} \lambda_{i} v_{j} \beta_{j} f=\sum_{j=0}^{n} v_{j}\left(x_{i}\right) \beta_{j} f, \quad i=0, \ldots, n,
$$

that is, $\left(\lambda_{0}, \ldots, \lambda_{n}\right)^{T}=M\left(\beta_{0}, \ldots, \beta_{n}\right)^{T}$. Multiplying by $M^{-1}$ and applying Proposition 2, the result follows.

The following theorem shows that the Lagrange representation, in terms of the evaluation functionals, has optimal conditioning. The conditioning of any other representation is greater than the conditioning of the Lagrange representation and the quotient can be bounded by the Skeel condition number of $M^{-1}$ where $M$ is the collocation matrix of the corresponding basis. Let us recall that the Skeel condition number of a nonsingular matrix $A$ is given by

$$
\operatorname{Cond}(A):=\left\|\left|A^{-1}\right||A|\right\|_{\infty} .
$$

Note that $\operatorname{Cond}(A) \geq 1$ for any nonsingular matrix $A$.

Theorem 4 Let $L[f]=\sum_{i=0}^{n} \beta_{i} f v_{i}$ be a representation of the Lagrange interpolation operator (1) and let $M=\left(v_{j}\left(x_{i}\right)\right)_{i, j=0, \ldots, n}$ be the collocation matrix of the basis $\left(v_{0}, \ldots, v_{n}\right)$. Then the following inequalities hold

$$
\operatorname{cond}(x ; \lambda) \leq \operatorname{cond}(x ; \beta) \leq \operatorname{Cond}\left(M^{-1}\right) \operatorname{cond}(x ; \lambda) .
$$

Proof: First, we have

$$
|L[f](x)| \leq\|f\|_{\infty} \sum_{i=0}^{n}\left\|\beta_{i}\right\|_{\infty}\left|v_{i}(x)\right|=\|f\|_{\infty} \operatorname{cond}(x ; \beta) .
$$

For each $x \in[a, b]$, we choose a function $f$ with $\|f\|_{\infty}=1$ such that $f\left(x_{i}\right)=$ $\operatorname{sign} l_{i}(x), i=0, \ldots, n$. Then, using formulae (4) and (5), we have

$$
\operatorname{cond}(x ; \lambda)=\lambda(x)=|L[f](x)| \leq \operatorname{cond}(x ; \beta) .
$$

Let $m_{i j}:=v_{j}\left(x_{i}\right)$ be the $(i, j)$ entry of the matrix $M$. By the Lagrange formula $v_{j}=\sum_{i=0}^{n} m_{i j} l_{i}$. Then we have

$$
\begin{aligned}
\operatorname{cond}(x ; \beta) & =\sum_{j=0}^{n}|| \beta_{j}\left\|_{\infty}\left|v_{j}(x)\right|=\sum_{j=0}^{n} \sum_{i=0}^{n}|| \beta_{j}\right\|_{\infty}\left|m_{i j}\right|\left|l_{i}(x)\right| \\
& \leq \operatorname{cond}(x ; \lambda) \max _{i=0, \ldots, n} \sum_{j=0}^{n}\left\|\beta_{j}\right\|_{\infty}\left|m_{i j}\right| .
\end{aligned}
$$


By Proposition 3 and by the definition of Skeel condition number

$$
\begin{aligned}
\max _{i=0, \ldots, n} \sum_{j=0}^{n}\left\|\beta_{j}\right\|_{\infty}\left|m_{i j}\right| & =\left\||M|\left(|| \beta_{0}\left\|_{\infty}, \ldots,\right\| \beta_{n} \|_{\infty}\right)^{T}\right\|_{\infty}=\left\||M|\left|M^{-1}\right| e\right\|_{\infty} \\
& =\left\||M|\left|M^{-1}\right|\right\|_{\infty}=\operatorname{Cond}\left(M^{-1}\right)
\end{aligned}
$$

and the result follows.

Theorem 4 shows that the ratio between the conditioning of a representation and the conditioning of the Lagrange representation is bounded above by Cond $\left(M^{-1}\right)$. The following result proves that this bound is attained at a node.

Theorem 5 Let $L[f]=\sum_{i=0}^{n} \beta_{i} f v_{i}$ be a representation of the Lagrange interpolation operator (1) on a subspace $U$ of $C[a, b]$ such that $1 \in U$. Then $\lambda(x) \geq 1$ for all $x \in[a, b]$, and

$$
\max _{x \in[a, b]} \frac{\operatorname{cond}(x ; \beta)}{\lambda(x)}=\max _{i=0, \ldots, n} \operatorname{cond}\left(x_{i} ; \beta\right)=\operatorname{Cond}\left(M^{-1}\right) .
$$

Proof: First, we show that $\lambda(x)$ does not vanish on $[a, b]$. Since $L[1]=1$, it follows that $\sum_{i=0}^{n} l_{i}(x)=1$ for all $x \in[a, b]$. Then, applying the triangular inequality, we have

$$
1 \leq \sum_{i=0}^{n}\left|l_{i}(x)\right|=\lambda(x)
$$

Since $\operatorname{cond}\left(x_{i} ; \beta\right)=\sum_{j=0}^{n}|| \beta_{j}\left\|_{\infty}\left|v_{j}\left(x_{i}\right)\right|=\sum_{j=0}^{n}|| \beta_{j}\right\|_{\infty}\left|m_{i j}\right|, i=0, \ldots, n$, we can use formula (6) to deduce

$$
\max _{i=0, \ldots, n} \operatorname{cond}\left(x_{i} ; \beta\right)=\max _{i=0, \ldots, n} \sum_{j=0}^{n}|| \beta_{j} \|_{\infty}\left|m_{i j}\right|=\operatorname{Cond}\left(M^{-1}\right) .
$$

Taking into account that $\lambda\left(x_{i}\right)=1, i=0, \ldots, n$, Theorem 4 and the previous formula, we have

$$
\begin{aligned}
\max _{i=0, \ldots, n} \operatorname{cond}\left(x_{i} ; \beta\right) & =\max _{i=0, \ldots, n} \frac{\operatorname{cond}\left(x_{i}, \beta\right)}{\lambda\left(x_{i}\right)} \leq \max _{x \in[a, b]} \frac{\operatorname{cond}(x, \beta)}{\lambda(x)} \\
& \leq \operatorname{Cond}\left(M^{-1}\right)=\max _{i=0, \ldots, n} \operatorname{cond}\left(x_{i} ; \beta\right) .
\end{aligned}
$$

So, all the above inequalities must be equalities and the result follows. 


\section{Stability of the Newton interpolation for- mula}

Let us now consider the case where $U=P_{n}$, the space of polynomials of degree less than or equal to $n$. In this case the Lagrange fundamental polynomials at the nodes $x_{0}, \ldots, x_{n}$ are

$$
l_{i}(x)=\prod_{j \in\{0, \ldots, n\} \backslash\{i\}} \frac{x-x_{j}}{x_{i}-x_{j}}, \quad i=0, \ldots, n .
$$

The Newton formula provides another representation of the Lagrange interpolation operator (1)

$$
L[f](x)=\sum_{i=0}^{n} d_{i} f \omega_{i}(x)
$$

in terms of the divided difference functionals

$$
d_{i} f:=\left[x_{0}, \ldots, x_{i}\right] f, \quad i=0, \ldots, n
$$

and the Newton basis $\left(\omega_{0}, \ldots, \omega_{n}\right)$, where

$$
\omega_{0}(x):=1, \quad \omega_{i}(x):=\left(x-x_{0}\right) \cdots\left(x-x_{i-1}\right), \quad i=1, \ldots, n+1 .
$$

Since the nodes are distinct we have

$$
d_{k} f=\sum_{i=0}^{k} \frac{f\left(x_{i}\right)}{\omega_{k+1}^{\prime}\left(x_{i}\right)}
$$

with $\omega_{k+1}^{\prime}\left(x_{i}\right)=\prod_{j \in\{0, \ldots, k\} \backslash\{i\}}\left(x_{i}-x_{j}\right)$ and, by Proposition 2,

$$
\left\|d_{k}\right\|_{\infty}=\sum_{j=0}^{k} \frac{1}{\left|\omega_{k+1}^{\prime}\left(x_{j}\right)\right|}=\sum_{j=0}^{k} \frac{1}{\prod_{l \in\{0, \ldots, k\} \backslash\{j\}}\left|x_{j}-x_{l}\right|} .
$$

By definition (2) we have

$$
\operatorname{cond}(x ; d)=\sum_{k=0}^{n} \sum_{j=0}^{k} \frac{\left|\omega_{k}(x)\right|}{\left|\omega_{k+1}^{\prime}\left(x_{j}\right)\right|}=\sum_{k=0}^{n} \sum_{j=0}^{k} \frac{\prod_{l \in\{0, \ldots, k-1\}}\left|x-x_{l}\right|}{\prod_{l \in\{0, \ldots, k\} \backslash\{j\}}\left|x_{j}-x_{l}\right|}
$$


Taking into account (9) and that $\omega_{k}\left(x_{i}\right)=0$, if $k>i$, we have

$$
\operatorname{cond}\left(x_{i} ; d\right)=\sum_{k=0}^{i}|| d_{k} \|_{\infty}\left|\omega_{k}\left(x_{i}\right)\right|=\sum_{k=0}^{i} \sum_{j=0}^{k} \frac{\left|\omega_{k}\left(x_{i}\right)\right|}{\left|\omega_{k+1}^{\prime}\left(x_{j}\right)\right|} .
$$

Applying Theorem 5 to the Newton representation and taking into account (10), we can derive the following result.

Corollary 6 Let $L[f]=\sum_{i=0}^{n} d_{i} f \omega_{i}$ be the Newton representation of the Lagrange polynomial operator (1) at the sequence of nodes $x_{0}, \ldots, x_{n}$. Then

$$
\begin{aligned}
& \max _{x \in[a, b]} \frac{\operatorname{cond}(x ; d)}{\lambda(x)}=\max _{i=0, \ldots, n} \operatorname{cond}\left(x_{i} ; d\right) \\
& =\max _{i=0, \ldots, n} \sum_{k=0}^{i} \sum_{j=0}^{k} \frac{\left|\omega_{k}\left(x_{i}\right)\right|}{\left|\omega_{k+1}^{\prime}\left(x_{j}\right)\right|}=\operatorname{Cond}\left(L^{-1}\right),
\end{aligned}
$$

where $L$ is the lower triangular matrix $L=\left(\omega_{j}\left(x_{i}\right)\right)_{0 \leq j \leq i \leq n}$.

\section{Leja order}

In [7] Leja introduced a sequence of points for a compact set in the complex plane satisfying remarkable properties of approximation of the Green function with a pole at infinity. Reichel [12] showed that the conditioning of the interpolation operator at the Leja points has subexponential growth. In his proof he uses the Newton formula showing that it has low conditioning. He also suggested that a Leja ordering, obtained by applying a Leja sequence to a finite set, has a nice behaviour with respect to the roundoff error propagation (see Section 5.3 of [5]).

Definition 1 A sequence of nodes $x_{0}, \ldots, x_{n}$ follows a Leja ordering if

$$
\left|\omega_{k}\left(x_{k}\right)\right| \geq\left|\omega_{k}\left(x_{i}\right)\right|, \quad 0 \leq k \leq i \leq n .
$$

A Leja order need not be unique. If we want to set the nodes in Leja order, we need to choose $x_{k}$ maximizing $\left|\omega_{k}(x)\right|$ on the set $x \in\left\{x_{k}, \ldots, x_{n}\right\}$. This fact suggests the following algorithm:

(i) First, we choose as the first node $x_{0}$ any node in the set. However, in order to maximize $\left|x_{1}-x_{0}\right|$ in the second step, we should choose one extreme point, either the minimum or the maximum. 
(ii) In the second step, $x_{1}$ is chosen such that

$$
\left|x_{1}-x_{0}\right|=\max _{j=1, \ldots, n}\left|x_{j}-x_{0}\right|
$$

So $x_{1}$ is the other extreme (minimum or maximum).

(iii) In the $k$-th step, we select $x_{k}$ such that

$$
\prod_{i=0}^{k-1}\left|x_{k}-x_{i}\right|=\max _{j=k, \ldots, n} \prod_{i=0}^{k-1}\left|x_{j}-x_{i}\right| .
$$

By formulae (10) and (11), we have that $\operatorname{cond}\left(x_{i} ; d\right) \leq \sum_{k=0}^{i}|| d_{k} \|_{\infty}\left|\omega_{k}\left(x_{k}\right)\right|$ and introducing the finite difference functionals

$$
\Delta\left(x_{0}, \ldots, x_{k}\right) f=\omega_{k}\left(x_{k}\right) d_{k} f, \quad k=0, \ldots, n,
$$

we deduce the following inequality for nodes in Leja ordering

$$
\operatorname{cond}\left(x_{i} ; d\right) \leq \sum_{k=0}^{i}\left\|\Delta\left(x_{0}, \ldots, x_{k}\right)\right\|_{\infty}
$$

The following result shows an upper bound of the norm of the finite difference functionals when the nodes follow a Leja ordering.

Theorem 7 Let $x_{0}, \ldots, x_{n}$ be nodes following a Leja ordering. Then

$$
\left\|\Delta\left(x_{0}, \ldots, x_{k}\right)\right\|_{\infty} \leq 2^{k}, \quad k=0, \ldots, n,
$$

and

$$
\max _{i=0, \ldots, n} \operatorname{cond}\left(x_{i} ; d\right) \leq 2^{n+1}-1 \text {. }
$$

Proof: By Proposition 3 applied to $L[f]=\sum_{k=0}^{n} \Delta\left(x_{0}, \ldots, x_{k}\right) \omega_{k}(x) / \omega_{k}\left(x_{k}\right)$, we have that

$$
\left(\begin{array}{c}
\left\|\Delta\left(x_{0}\right)\right\|_{\infty} \\
\vdots \\
\left\|\Delta\left(x_{0}, \ldots, x_{n}\right)\right\|_{\infty}
\end{array}\right)=\left|\tilde{L}^{-1}\right| e,
$$

where $\tilde{L}$ is the lower triangular matrix with unit diagonal whose entries are $\tilde{l}_{i j}=\omega_{j}\left(x_{i}\right) / \omega_{j}\left(x_{j}\right)$ and $e=(1, \ldots, 1)^{T}$. Let $s$ be a vector whose components 
satisfy $\|s\|_{\infty} \leq 1$. Let $c=\tilde{L}^{-1} s$ and let us show by induction on $k$ that $\left|c_{k}\right| \leq 2^{k}$, for all $k=0, \ldots, n$. For $k=0, c_{0}=s_{0}$ and the inequality $\left|c_{0}\right| \leq 1$ follows. Assuming that the inequality holds for $k-1$, let us prove it for $k$.

Since the nodes follow a Leja order, we deduce that

$$
\left|\tilde{l}_{i j}\right| \leq 1, \quad j \leq i
$$

Taking into account that

$$
c_{k}=s_{k}+\sum_{j=0}^{k-1} \tilde{l}_{k j} c_{j}
$$

we have

$$
\left|c_{k}\right| \leq\left|s_{k}\right|+\sum_{j=0}^{k-1}\left|\tilde{l}_{k j}\right|\left|c_{j}\right| \leq 1+\left(1+2+\cdots+2^{k-1}\right)=2^{k} .
$$

Take $s=\left(\operatorname{sign}\left(\tilde{l}_{k j}^{(-1)}\right)\right)_{j=0, \ldots, n}$. Then, by formula $(13)$,

$$
\left\|\Delta\left(x_{0}, \ldots, x_{k}\right)\right\|_{\infty}=\sum_{j=0}^{k}\left|\widetilde{l}_{k j}^{(-1)}\right|=\sum_{j=0}^{k} \tilde{l}_{k j}^{(-1)} \operatorname{sign}\left(\widetilde{l}_{k j}^{(-1)}\right) \leq 2^{k}
$$

From formula (12) it follows cond $\left(x_{i} ; d\right) \leq 2^{i+1}-1$ and the result follows.

Using the previous result and Theorem 5 we deduce that the ratio between the conditioning of the Newton representation with nodes following a Leja ordering and the conditioning of the Lagrange representation is bounded above by $2^{n+1}-1$. In Section 7 , we will see that the conditioning at equidistant nodes in a Leja ordering is much lower than the bound $2^{n+1}-1$.

\section{Case of equidistant nodes}

From now on, we shall consider equidistant nodes $x_{0}, \ldots, x_{n}$ in $[a, b]$

$$
x_{i}=a+i h, \quad i=0, \ldots, n, \text { with } h=\frac{b-a}{n},
$$

and apply the previous results to derive the conditioning of the Newton formula. In order to compare it with the conditioning of the Lagrange formula, 
we use some known facts on the Lebesgue constant $\Lambda_{n}$ at equidistant nodes. In [14] Schönhage shows the following asymptotic formula

$$
\Lambda_{n} \sim \frac{2^{n+1}}{e n \log (n+\gamma)}
$$

where $\gamma$ is the Euler-Mascheroni constant. We shall use the following bound

$$
\lambda(x) \leq 2^{n}, \quad x \in[a, b]
$$

based on a private communication by Jia Rong-Qing to Carl de Boor (see 1.3.22 of [13]). In order to bound $\operatorname{cond}(x ; d)$ associated with the Newton representation, we need the following auxiliary result.

Lemma 8 Let $x_{i}=a+\frac{i}{n}(b-a), i=0, \ldots, n$, and let $d_{k}, k=0, \ldots, n$ and $\omega_{k}, k=0, \ldots, n+1$, be defined by (7) and (8), respectively. Then

(i) $\omega_{k}\left(x_{i}\right)=\left(\frac{b-a}{n}\right)^{k} \frac{i !}{(i-k) !}, 0 \leq k \leq i \leq n$,

(ii) $\omega_{k+1}^{\prime}\left(x_{i}\right)=(-1)^{k-i}\left(\frac{b-a}{n}\right)^{k} i !(k-i) !, 0 \leq i \leq k \leq n$,

(iii) $\left\|d_{k}\right\|_{\infty}=\frac{1}{k !}\left(\frac{b-a}{n}\right)^{-k} 2^{k}, 0 \leq k \leq n$.

Proof: In order to prove (i), let us observe that

$$
\omega_{k}\left(x_{i}\right)=\prod_{j=0}^{k-1}\left(x_{i}-x_{j}\right)=\left(\frac{b-a}{n}\right)^{k} \prod_{j=0}^{k-1}(i-j)=\left(\frac{b-a}{n}\right)^{k} \frac{i !}{(i-k) !} .
$$

Now we show (ii):

$$
\omega_{k+1}^{\prime}\left(x_{i}\right)=\prod_{j \neq i}\left(x_{i}-x_{j}\right)=\left(\frac{b-a}{n}\right)^{k} \prod_{j \neq i}(i-j)=(-1)^{k-i}\left(\frac{b-a}{n}\right)^{k} i !(k-i) !
$$

Finally,

$$
\left\|d_{k}\right\|_{\infty}=\sum_{j=0}^{k} \frac{1}{\left|\omega_{k+1}^{\prime}\left(x_{j}\right)\right|}=\left(\frac{b-a}{n}\right)^{-k} \sum_{j=0}^{k} \frac{1}{j !(k-j) !}=\frac{1}{k !}\left(\frac{b-a}{n}\right)^{-k} \sum_{j=0}^{k}\left(\begin{array}{l}
k \\
j
\end{array}\right)
$$


and the result follows.

From Lemma 8 (i), we deduce that the entries of the lower triangular collocation matrix $L=\left(\omega_{j}\left(x_{i}\right)\right)_{i, j=0, \ldots, n}$ are

$$
l_{i j}=\omega_{j}\left(x_{i}\right)=\left(\frac{b-a}{n}\right)^{j} \frac{i !}{(i-j) !}=\left(\frac{b-a}{n}\right)^{j} j !\left(\begin{array}{l}
i \\
j
\end{array}\right), \quad 0 \leq j \leq i \leq n .
$$

The following result provides $\operatorname{Cond}\left(L^{-1}\right)=\max _{i=0, \ldots, n} \operatorname{cond}\left(x_{i} ; d\right)$ for equidistant nodes.

Theorem 9 Let $L[f]=\sum_{i=0}^{n} d_{i} f \omega_{i}$ be the Newton representation of the Lagrange polynomial operator (1) at the sequence of equidistant nodes $x_{i}=$ $a+\frac{i}{n}(b-a), i=0, \ldots, n$. Then $\operatorname{cond}\left(x_{i} ; d\right)=3^{i}, i=0, \ldots, n$, and so

$$
\max _{i=0, \ldots, n} \operatorname{cond}\left(x_{i} ; d\right)=\operatorname{cond}\left(x_{n} ; d\right)=3^{n} .
$$

Proof: Taking into account $\omega_{k}\left(x_{i}\right)=0$ if $k>i$, we have

$$
\operatorname{cond}\left(x_{i} ; d\right)=\sum_{k=0}^{i}\left\|d_{k}\right\|_{\infty}\left|\omega_{k}\left(x_{i}\right)\right| .
$$

Using Lemma 8,

$$
\operatorname{cond}\left(x_{i} ; d\right)=\sum_{k=0}^{i} \frac{i !}{(i-k) !} 2^{k}=\sum_{k=0}^{i}\left(\begin{array}{l}
i \\
k
\end{array}\right) 2^{k}=3^{i} .
$$

The previous result shows that the evaluation of the Newton formula for equidistant nodes can be less stable than the evaluation of the Lagrange formula. The Lebesgue function takes values close to 1 in a neighborhood of each node. In contrast, $\operatorname{cond}(x ; d)$ takes the value $3^{i}$ at the node $x_{i}$, giving rise to a significant loss of stability at the nodes. Corollary 6 and Theorem 9 show that the maximum value of $\operatorname{cond}(x ; d) / \lambda(x)$ is attained at the last node $x_{n}$. Therefore, for the remaining points,

$$
\operatorname{cond}(x ; d) \leq 3^{n} \lambda(x),
$$

and using the bound (15), we deduce the uniform bound $\operatorname{cond}(x ; d) \leq 6^{n}$. This bound is too pessimistic. In the next section we show that $\operatorname{cond}(x ; d) \leq$ $3^{n}$ for all $x \in[a, b]$. 


\section{Optimal bound for the stability of the New- ton formula for equidistant nodes}

In order to bound $\operatorname{cond}(x ; d)$ for the Newton formula at equidistant nodes

(14), we make the change of variables $x=a+t h$, which transforms the nodes into the first nonnegative integers $0,1, \ldots, n$. We observe that

$$
\omega_{k}(a+t h)=h^{k} t(t-1) \cdots(t-k+1) .
$$

We can show the following result.

Lemma 10 Let $i$ be an integer such that $1 \leq i \leq n$ and $\omega_{k}$ given by (16). Then, for each $t \in[i-1, i]$, we have

$$
\begin{aligned}
& \left|\omega_{k}(a+t h)\right| \leq h^{k} \frac{i !}{(i-k) !}, \quad \text { if } 0 \leq k \leq i, \\
& \left|\omega_{k}(a+t h)\right| \leq \frac{h^{k}}{4} i !(k-i) !, \quad \text { if } i<k \leq n .
\end{aligned}
$$

Proof: If $k \leq i$, we deduce, from the fact that the function $t(t-1) \cdots(t-$ $k+1)$ is a nondecreasing function on $[k-1, \infty)$, that $\omega_{k}(a+t h) \leq \omega_{k}(a+i h)$. Then

$$
\left|\omega_{k}(a+t h)\right| \leq h^{k} i(i-1) \cdots(i-k+1)=h^{k} \frac{i !}{(i-k) !} .
$$

If $k \geq i+1$, we have that $[i-1, i] \subseteq[0, k-1]$ and

$$
\left|\omega_{k}(a+t h)\right|=h^{k}|t| \cdots|t-i+2||t-i+1||t-i||t-i-1| \cdots|t-k+1| .
$$

We now use the following bounds to deduce the results

$$
\begin{aligned}
& |t-i+1||t-i| \leq \frac{1}{4}, \\
& |t||t-1| \cdots|t-i+2| \leq i(i-1) \cdots 2=i ! \\
& |t-i-1| \cdots|t-k+1| \leq 2 \cdots(k-i)=(k-i) !
\end{aligned}
$$

We provide a bound for $\operatorname{cond}(x ; d)$ in the next theorem. 
Theorem 11 Let $x_{0}, \ldots, x_{n}$ be equidistant nodes in $[a, b]$ given by (14). Let $i$ be an integer such that $1 \leq i \leq n$. Then

$$
\operatorname{cond}(x ; d) \leq 3^{i}+\frac{1}{4} \sum_{k=i+1}^{n} \frac{2^{k}}{k}, \quad x \in\left[x_{i-1}, x_{i}\right] .
$$

Proof: We have that $x=a+t h$ with $t \in[i-1, i]$ and $h=(b-a) / n$. By Corollary 6, we have

$$
\operatorname{cond}(x ; d)=\sum_{k=0}^{n} \sum_{j=0}^{k} \frac{\left|\omega_{k}(x)\right|}{\left|\omega_{k+1}^{\prime}\left(x_{j}\right)\right|}=\sum_{k=0}^{i} \sum_{j=0}^{k} \frac{\left|\omega_{k}(x)\right|}{\left|\omega_{k+1}^{\prime}\left(x_{j}\right)\right|}+\sum_{k=i+1}^{n} \sum_{j=0}^{k} \frac{\left|\omega_{k}(x)\right|}{\left|\omega_{k+1}^{\prime}\left(x_{j}\right)\right|} .
$$

We apply Lemma 8 (ii) and Lemma 10 to bound both terms. For the first term

$$
\begin{aligned}
& \sum_{k=0}^{i} \sum_{j=0}^{k} \frac{\left|\omega_{k}(x)\right|}{\left|\omega_{k+1}^{\prime}\left(x_{j}\right)\right|} \leq \sum_{k=0}^{i} \sum_{j=0}^{k} \frac{h^{k} i ! /(i-k) !}{h^{k} j !(k-j) !} \\
& =\sum_{k=0}^{i} \sum_{j=0}^{k}\left(\begin{array}{l}
i \\
k
\end{array}\right)\left(\begin{array}{l}
k \\
j
\end{array}\right)=\sum_{k=0}^{i}\left(\begin{array}{l}
i \\
k
\end{array}\right) 2^{k}=3^{i},
\end{aligned}
$$

and for the second one

$$
\begin{aligned}
\sum_{k=i+1}^{n} \sum_{j=0}^{k} \frac{\left|\omega_{k}(x)\right|}{\left|\omega_{k+1}^{\prime}\left(x_{j}\right)\right|} & \leq \frac{1}{4} \sum_{k=i+1}^{n} \sum_{j=0}^{k} \frac{h^{k} i !(k-i) !}{h^{k} j !(k-j) !}=\frac{1}{4} \sum_{k=i+1}^{n} \sum_{j=0}^{k} \frac{\left(\begin{array}{l}
k \\
j
\end{array}\right)}{\left(\begin{array}{c}
k \\
i
\end{array}\right)} \\
& =\frac{1}{4} \sum_{k=i+1}^{n} \frac{2^{k}}{\left(\begin{array}{c}
k \\
i
\end{array}\right)} .
\end{aligned}
$$

Since $k>i \geq 1$, we can deduce that $\left(\begin{array}{l}k \\ i\end{array}\right) \geq k$ and hence

$$
\frac{1}{4} \sum_{k=i+1}^{n} \frac{2^{k}}{\left(\begin{array}{c}
k \\
i
\end{array}\right)} \leq \frac{1}{4} \sum_{k=i+1}^{n} \frac{2^{k}}{k}
$$

Then the result follows.

We finish by showing that $3^{n}$ is the best uniform bound for $\operatorname{cond}(x ; d)$ and it is attained at $x_{n}$. 
Corollary 12 Let $x_{0}, \ldots, x_{n}$ be the equidistant nodes in $[a, b]$ given by (14) and $x \in[a, b]$. Then

$$
\max _{x \in[a, b]} \operatorname{cond}(x ; d)=\operatorname{cond}\left(x_{n} ; d\right)=3^{n} .
$$

Proof: Let us first show that the sequence $f_{i}:=3^{i}+2^{n-1}-2^{i-1}, i=1, \ldots, n$, is nondecreasing. In fact,

$$
\Delta f_{i}=f_{i+1}-f_{i}=3^{i+1}+2^{n-1}-2^{i}-3^{i}-2^{n-1}+2^{i-1}=2 \cdot 3^{i}-2^{i-1} \geq 0 .
$$

Then, for each $x$ there exists an integer $i, 1 \leq i \leq n$, such that $x \in\left[x_{i-1}, x_{i}\right]$ and, applying Theorem 11, we have

$$
\operatorname{cond}(x ; d) \leq 3^{i}+\frac{1}{4} \sum_{k=i+1}^{n} \frac{2^{k}}{k} \leq 3^{i}+\frac{1}{4} \sum_{k=i+1}^{n} 2^{k}=3^{i}+2^{n-1}-2^{i-1}=f_{i} .
$$

Since $f_{i} \leq f_{n}=3^{n}$, the result holds applying Theorem 9 .

Let us remark that $\operatorname{cond}(x ; d)$ is invariant under affine transformations $T(x)=r x+s, r \neq 0$. Let

$$
T^{*}\left(d_{k}\right)=\left[T\left(x_{0}\right), \ldots, T\left(x_{k}\right)\right]
$$

be the divided differences functionals at the set of transformed nodes. Then

we deduce from formula $(9)$ that $\operatorname{cond}(x ; d)=\operatorname{cond}\left(T(x), T^{*}(d)\right)$. Taking $T(x)=a+b-x$, we can deduce analogous results for decreasing nodes. In fact, Theorem 9 and Corollary 12 also hold and Theorem 11 transforms into

$$
\operatorname{cond}(x ; d) \leq 3^{i}+\frac{1}{4} \sum_{k=i+1}^{n} \frac{2^{k}}{k}, \quad x \in\left[x_{i}, x_{i-1}\right] .
$$

Observe that, in this case, the last node is $x_{n}=a$, the left end of the interval.

\section{Numerical experiments}

In this section we provide some numerical examples to illustrate results of the previous sections. First, let us interprete Theorem 11 and Corollary 12 using formula (3). Due to roundoff errors, we can assume that the data come from $\hat{f}$, a perturbation of $f$, such that

$$
\|f-\hat{f}\|_{\infty} \leq u\|f\|_{\infty},
$$


where $u$ is the unit roundoff. Then the error in the computation of the interpolant can be bounded above by

$$
|L[f](x)-L[\hat{f}](x)| \leq u \operatorname{cond}(x ; d)\|f\|_{\infty} \leq 3^{n} u\|f\|_{\infty},
$$

for the Newton representation with equidistant nodes. If $3^{n} \leq u^{-1}$ then the roundoff error will be less than $\|f\|_{\infty}$. Therefore, the Newton formula can have roundoff errors greater than $\|f\|_{\infty}$ only if $n \geq 24 \log (2) / \log (3) \approx 15.14$, for single precision arithmetic $\left(u^{-1}=2^{24}\right)$, or $n \geq 53 \log (2) / \log (3) \approx 33.44$, for double precision arithmetic $\left(u^{-1}=2^{53}\right)$. The Lebesgue constant takes values greater than the inverse of the roundoff unit if $n \geq 32$, for single precision, or $n \geq 62$, for double precision. Therefore, for double precision arithmetic and degrees between 33 and 62, the relative errors of the Lagrange formula are less than 1 whereas the Newton formula might gives relative errors greater than 1, especially when the point $x$ is close to the last node $x_{n}$.

For the numerical experiments, we have considered the interpolation data

$$
\begin{aligned}
& x_{i}=-1+\frac{2 i}{n}, \quad i=0, \ldots, n, \\
& f\left(x_{0}\right)=2, \quad f\left(x_{i}\right)=1, \quad i=1, \ldots, n .
\end{aligned}
$$

The polynomial interpolant is

$$
p(x)=1+\frac{\left(x_{1}-x\right) \cdots\left(x_{n}-x\right)}{\left(1+x_{1}\right) \cdots\left(1+x_{n}\right)} .
$$

We have tested the Newton formula with different degrees and found that the computations give errors close to 1 for $n=54$, a much higher degree than 34 , the degree predicted with the bound $3^{n}$. Figure 1 shows that the decimal logarithm of the absolute error due to rounding in the Newton formula grows linearly, confirming the exponential growth of the conditioning predicted by Theorem 11. The maximum of the error is achived near $x_{n}=1$, the last interpolation point, which agrees with the fact that the conditioning takes its maximum at $x_{n}$, as stated in Corollary 12 .

We end by comparing equidistant nodes in increasing order and in a Leja order. We illustrate graphically Theorem 11 in Figure 2, which depicts $\log _{3} \operatorname{cond}(x ; d)$ at equidistant nodes in increasing order and with a Leja ordering and $\log _{3} \lambda(x)$ for $n=10$ in the interval $[0,1]$. The conditioning for 


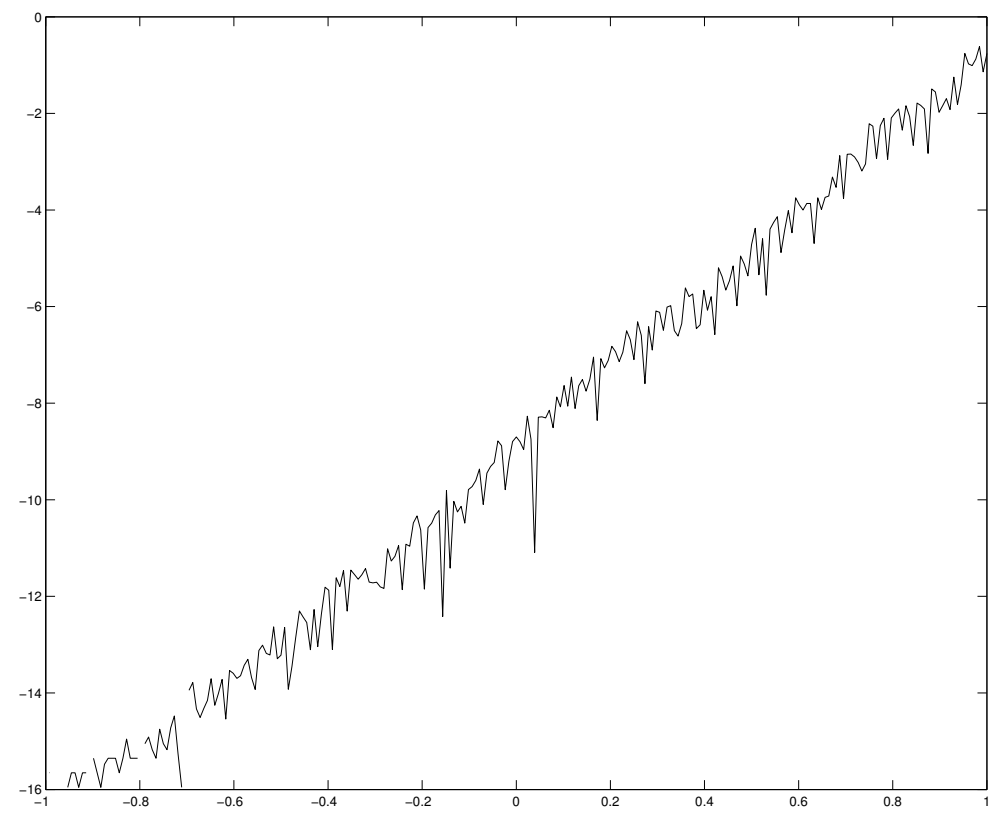

Figure 1: Decimal logarithm of roundoff error of Newton formula for equidistant points in $[-1,1]$ with degree $n=54$.

nodes following a Leja ordering is closer to the Lebesgue constant than the conditioning for increasing order.

Although, for an arbitrary set of $n+1$ nodes in a Leja order, we have seen in Theorem 7 that cond $(x ; d)$ is bounded above by $2^{n+1}-1$, this bound is very pessimistic for equidistant nodes in a Leja order. Figure 3 compares the graphics of $n \log n$ with the maximum of the conditioning for equidistant nodes following a Leja order. Finally, Table 1 compares $\max _{i} \operatorname{cond}\left(x_{i} ; d\right)$ at equidistant nodes with increasing order and with a Leja order.

\section{References}

[1] Berrut, J.P., Trefethen, L.N., Barycentric Lagrange interpolation, SIAM Rev. 46, 501-517 (2004). 


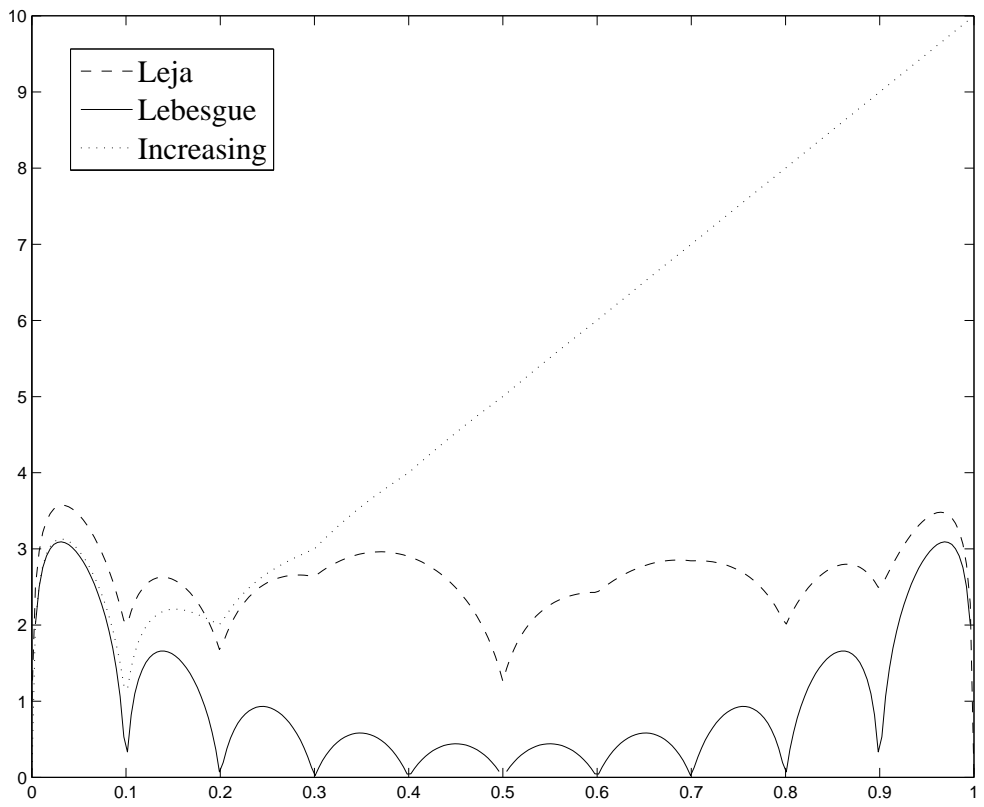

Figure 2: $\log _{3}(\lambda(x))$ and $\log _{3}(\operatorname{cond}(x ; d))$ for nodes in increasing order and with a Leja order.

\begin{tabular}{|c|c|c|}
\hline $\mathrm{n}$ & Increasing & Leja \\
\hline 3 & 27 & 6.3333 \\
\hline 4 & 81 & 9.25 \\
\hline 5 & $2.43 \times 10^{2}$ & 10.3 \\
\hline 9 & $1.9683 \times 10^{4}$ & 23.8833 \\
\hline 19 & $1.1623 \times 10^{9}$ & 50.9247 \\
\hline 29 & $6.8630 \times 10^{13}$ & 75.9233 \\
\hline 39 & $4.0525 \times 10^{18}$ & $1.1567 \times 10^{2}$ \\
\hline
\end{tabular}

Table 1: $\max _{i} \operatorname{cond}\left(x_{i} ; d\right)$ at equidistant nodes in increasing order and in a Leja order 


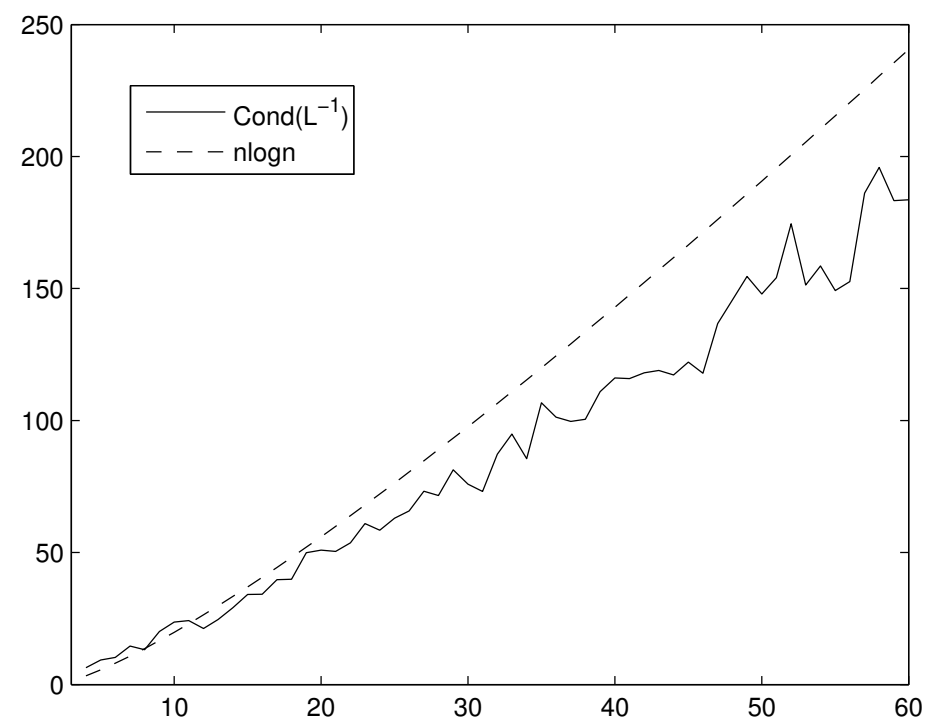

Figure 3: Comparison between $\operatorname{Cond}\left(L^{-1}\right)=\max _{i} \operatorname{cond}\left(x_{i} ; d\right)$ for equidistant nodes in a Leja order and $n \log n$ for degrees from $n=3$ to $n=60$.

[2] Cheney, W., Light, W., A course in Approximation Theory. Brook/Cole Publishing Company, Pacific Grove, CA. (2000).

[3] Farouki, R.T., Goodman, T.N.T., On the optimal stability of the Bernstein basis, Math. Comp., 65, 1553-1566 (1996).

[4] Farouki, R.T., Rajan, V.T., On the numerical condition of algebraic curves and surfaces 1. Implicit equations, Computer Aided Geometric Design, 5, 215-252 (1988).

[5] Higham, N.J., Accuracy and Stability of Numerical Algorithms. SIAM, Second Edition (2002).

[6] Higham, N.J., The numerical stability of barycentric Lagrange interpolation, IMA J. Numer. Anal., 24, 547-556 (2004).

[7] Leja, F., Sur certaines suites liées aux ensembles plans et leur application à la représentation conforme, Ann. Polon. Math., 4, 8-13 (1957). 
[8] Lyche, T., Peña, J.M., Optimally stable multivariate bases, Advances in Computational Mathematics, 20, 149-159 (2004).

[9] Peña, J.M., B-splines and optimal stability, Math. Comp., 66, 1555-1560 (1997).

[10] Peña, J.M., On the optimal stability of bases of univariate functions, Numer. Math., 91, 305-318 (2002).

[11] Peña, J.M., A note on the optimal stability of bases of univariate functions, Numer. Math., 103, 151-154 (2006).

[12] Reichel, L., Newton interpolation at Leja points, BIT 30, 2, 332-346 (1990).

[13] Rivlin, T.J., Chebyshev polynomials. From approximation theory to algebra and number theory. Pure and Applied Mathematics (New York). John Wiley \& Sons, Inc., New York (1990).

[14] Schönhage, A., Fehlerfortpflanzung bei Interpolation, Numer. Math., 3, 62-71 (1961).

[15] Trefethen, L.N., Six myths of polynomial interpolation and quadrature, Math. Today (Southend-on-Sea), 47, 184-188 (2011).

[16] Trefethen, L.N., Approximation theory and approximation practice. Society for Industrial and Applied Mathematics. SIAM, Philadelphia, PA (2013). 